\title{
On a Problem of Finbarr Holland
}

\author{
Mohamed AkKouchi
}

\section{Introduction}

1.1 In his article $[\mathrm{H}]$, Finbarr Holland has solved and discussed a problem that the students were given on the first morning of the 1999 IMO in Bucharest. They were asked to find, for all integers $n \geq 2$, the smallest constant $C$ such that

$$
\sum_{1 \leq i<j \leq n} x_{i} x_{j}\left(x_{i}^{2}+x_{j}^{2}\right) \leq C\left(\sum_{i=1}^{n} x_{i}\right)^{4},
$$

for all non-negative real numbers $x_{1}, x_{2}, \ldots, x_{n}$, and to specify when equality occurs for this value of $C$. At the end of $[\mathrm{H}]$, Finbarr Holland proposed the following problem.

1.2 Problem. Let $n \geq 2$ be a positive integer and $p$ a positive number. Determine (a) the best constant $C_{p, n}$ such that

$$
\sum_{1 \leq i<j \leq n} x_{i} x_{j}\left(x_{i}^{p}+x_{j}^{p}\right) \leq C_{p, n}\left(\sum_{i=1}^{n} x_{i}\right)^{p+2}
$$

for all non-negative real numbers $x_{1}, x_{2}, \ldots, x_{n}$, and (b) the cases of equality.

The object of this note is to propose a solution to this problem. Precisely we shall prove the following the result. 
1.3 Proposition. Let $n \geq 2$ be a positive integer and $p$ a positive real number. Then the following assertions hold true.

(a) The best constant $C_{p, n}$ satisfying (1) depends only on $p$ and satisfies $\frac{1}{2^{p+1}} \leq C_{p, 2}=C_{p, n}$ for all integers $n \geq 2$.

(b) For all $p>0$, we have $C_{p, 2}=\max \left\{(1-t) t^{p+1}+t(1-t)^{p+1}\right.$ : $\left.0 \leq t \leq \frac{1}{2}\right\}$.

(c) If $p \geq 2$, then $C_{p, 2}=\frac{p^{p}}{(p+1)^{p+1}}$.

(d) If $1<p<2$, then $\frac{1}{2^{p+1}} \leq C_{p, 2} \leq \frac{1}{2^{p+2}}+\frac{(p+1)^{p+1}}{(p+2)^{p+2}}$.

(e) If $0<p \leq 1$, then $C_{p, 2}=\frac{p^{p}}{(p+1)^{p+1}}$.

\section{Proofs}

We start by giving an equivalent formulation of Problem 1.2.

2.1 Problem. Let $n \geq 2$ be a positive integer and $p>0$. Determine (a) the smallest constant $C_{p, n}$ such that

$$
\sum_{i=1}^{n} x_{i}^{p+1}\left(1-x_{i}\right) \leq C_{p, n}
$$

for all non-negative real numbers $x_{1}, x_{2}, \ldots, x_{n}$ satisfying $\sum_{i=1}^{n} x_{i}=$ 1 , and (b) the cases of equality.

This formulation holds since we have the following equality:

$$
\sum_{1 \leq i<j \leq n} x_{i} x_{j}\left(x_{i}^{p}+x_{j}^{p}\right)=\left(\sum_{i=1}^{n} x_{i}\right)\left(\sum_{i=1}^{n} x_{i}^{p+1}\right)-\sum_{i=1}^{n} x_{i}^{p+2} .
$$

We let $\Delta_{n}:=\left\{\left(x_{1}, x_{2}, \ldots, x_{n}\right) \in[0,1]^{n}: \sum_{i=1}^{n} x_{i}=1\right\}$. For every real number $t$, we set $F_{p}(t):=(1-t) t^{p}$. For all $x=\left(x_{1}, x_{2}, \ldots, x_{n}\right) \in$ $\mathbb{R}^{n}$, we put $H_{p}^{n}(x):=\sum_{i=1}^{n} x_{i} F_{p}\left(x_{i}\right)$. Another formulation of Problem 2.1 is then as follows.

2.2 Problem. Let $n \geq 2$ be a positive integer and $p>0$. Determine (a) $\sup \left\{H_{p}^{n}(x): x \in \bar{\Delta}_{n}\right\}=C_{p, n}$, and (b) the set of points in $\Delta_{n}$ where this maximum is attained.

2.3. We start with the case $p \geq 1$. Along the lines of the paper $[\mathrm{H}]$, we distinguish four cases; Case (A), in which we suppose all of the $x_{i}$ 
are in the interval $\left[0, \frac{1}{2}[\right.$; Case (B) in which we suppose that exactly one of the $x_{i}$ belongs to $\left.] \frac{1}{2}, 1\right]$; Case $(\mathrm{C})$ in which we suppose that exactly one of the $x_{i}$ equals to $\frac{1}{2}$; and Case (D) in which we suppose that precisely two of the $x_{i}$ are equal to $\frac{1}{2}$. These cases exhaust all possibilities.

Case (A). It is easy to see that the function $x \mapsto x^{p}(1-x)$ is strictly increasing on the interval $\left[0, \frac{1}{2}\left[\right.\right.$, and that $\max \left\{x^{p}(1-x): 0 \leq x<\right.$ $\left.\frac{1}{2}\right\}=\frac{1}{2^{p+1}}$. So, in this case, we have

$$
\begin{aligned}
\sum_{i=1}^{n} x_{i}^{p+1}\left(1-x_{i}\right) & \leq \max _{1 \leq i \leq n} x_{i}^{p}\left(1-x_{i}\right) \sum_{i=1}^{n} x_{i} \\
& =\max _{1 \leq i \leq n} x_{i}^{p}\left(1-x_{i}\right) \\
& <\frac{1}{2^{p+1}} .
\end{aligned}
$$

Case (B). Suppose that $x_{k}$ belongs to $\left.] \frac{1}{2}, 1\right]$. We denote it by $a$ and relabel the remaining $n-1$ variables as $y_{1}, y_{2}, \ldots, y_{n-1}$. We have the relation $1-a=\sum_{i=1}^{n-1} y_{i}$, from which we deduce that each $y_{i}$ belongs to $[0,1-a] \subset\left[0, \frac{1}{2}[\right.$. It follows that

$$
y_{i}^{p}\left(1-y_{i}\right) \leq a(1-a)^{p}, i=1,2, \ldots, n-1 .
$$

Hence

$$
\begin{aligned}
\sum_{i=1}^{n} x_{i}^{p+1}\left(1-x_{i}\right) & =a^{p+1}(1-a)+\sum_{i=1}^{n-1} y_{i}^{p+1}\left(1-y_{i}\right) \\
& \leq a^{p+1}(1-a)+\max _{1 \leq i \leq n-1} y_{i}^{p}\left(1-y_{i}\right) \sum_{i=1}^{n-1} y_{i} \\
& \leq(1-a) a^{p+1}+a(1-a)^{p+1} .
\end{aligned}
$$

We conclude that in this case, we have

$$
\sum_{i=1}^{n} x_{i}^{p+1}\left(1-x_{i}\right) \leq \max \left\{(1-t) t^{p+1}+t(1-t)^{p+1}: \frac{1}{2} \leq t \leq 1\right\} .
$$

Case (C). This is treated in a similar manner to Case (B), and we obtain

$$
\sum_{i=1}^{n} x_{i}^{p+1}\left(1-x_{i}\right) \leq \frac{1}{2^{p+1}}
$$


Case (D) is trivial, and it shows that we have $C_{p, n} \geq \frac{1}{2^{p+1}}$.

The conclusion of this analysis so far is that, if $x_{1}, x_{2}, \ldots, x_{n}$ are non-negative numbers satisfying $\sum_{i=1}^{n} x_{i}=1$, then

$$
\sum_{i=1}^{n} x_{i}^{p+1}\left(1-x_{i}\right) \leq \max \left\{(1-t) t^{p+1}+t(1-t)^{p+1}: \frac{1}{2} \leq t \leq 1\right\} .
$$

It is clear that

$$
C_{p, 2}=\max \left\{(1-t) t^{p+1}+t(1-t)^{p+1}: \frac{1}{2} \leq t \leq 1\right\},
$$

and it is easy to see that $C_{p, 2}$ at most is

$$
C_{p, n}=\max \left\{\sum_{i=1}^{n} x_{i}^{p+1}\left(1-x_{i}\right): x_{1}, \ldots, x_{n} \in[0,1], \sum_{i=1}^{n} x_{i}=1\right\} .
$$

Thus, we have proved part of (a). The assertion (b) is clear.

2.4. We suppose that $p>0$. Let us introduce the function

$$
h_{p}(t):=H_{p}^{2}(t, 1-t)=t(1-t)\left[t^{p}+(1-t)^{p}\right] \quad \forall t \in \mathbb{R} .
$$

Since the function $F_{p}$ is concave on $\left[0, \infty\left[\right.\right.$ having $F_{p}\left(\frac{p}{p+1}\right)$ as the (only) maximal value, we obtain, for all $t \in[0,1]$,

$$
\begin{aligned}
h_{p}(t) & =t F_{p}(t)+(1-t) F_{p}(t) \\
& \leq F_{p}\left(t^{2}+(1-t)^{2}\right) \leq F_{p}\left(\frac{p}{p+1}\right)=\frac{p^{p}}{(p+1)^{p+1}} .
\end{aligned}
$$

Since $h_{p}\left(\frac{1}{2}\right)=\frac{1}{2^{p+1}}$, we deduce that $\frac{1}{2^{p+1}} \leq C_{p, 2} \leq \frac{p^{p}}{(p+1)^{p+1}}$ for all $p>0$.

2.5. We suppose that $p \in\left[2, \infty\left[\right.\right.$. We shall prove that $C_{p, 2}=$ $\frac{p^{p}}{(p+1)^{p+1}}$. To this end, we consider $u:=\frac{1}{2}\left(1-\sqrt{\frac{p-1}{p+1}}\right)$ and $v:=\frac{1}{2}(1+$ $\sqrt{\frac{p-1}{p+1}}$ ); these are the solutions (belonging to $[0,1]$ ) of the algebraic equation $t^{2}+(1-t)^{2}=\frac{p}{p+1}$. According to the convexity of the function $t \mapsto t^{p-1}$ on $[0, \infty[$, we deduce that

$$
\begin{aligned}
h_{p}(u) & =\frac{p}{2(p+1)}\left(u u^{p-1}+v v^{p-1}\right) \\
& \geq \frac{p}{2(p+1)}\left(u^{2}+v^{2}\right)^{p-1}=\frac{p^{p}}{2(p+1)^{p}} \geq \frac{p^{p}}{(p+1)^{p+1}} .
\end{aligned}
$$


According to 2.5 , we conclude that $C_{p, 2}=\frac{p^{p}}{(p+1)^{p+1}}$.. Thus we have proved (c). The assertion (d) is easy to obtain, hence we omit it.

2.6. To complete the proof, we look at the case $0<p \leq 1$. It is clear that in this case, the function $t \mapsto t^{p-1}$ is decreasing and convex on $] 0, \infty[$. For all $\left.t \in] 0, \frac{p}{(p+1)}\right]$, we have $1-t \geq \frac{1}{p+1}$ and $t^{2}+(1-t)^{2} \geq \frac{1}{2} \geq \frac{p}{p+1}$, therefore

$$
\begin{aligned}
C_{p, 2} & \geq h_{p}(t)=t(1-t)\left(t t^{p-1}+(1-t)(1-t)^{p-1}\right) \\
& \geq t(1-t)\left(t^{2}+(1-t)^{2}\right)^{p-1} \geq t \frac{p^{p-1}}{(p+1)^{p}} .
\end{aligned}
$$

We deduce then that $C_{p, 2} \geq \frac{p^{p}}{(p+1)^{p+1}}$. For every integer $n \geq 2$ and all $x \in \Delta$, we have

$$
\sum_{i=1}^{n} x_{i}^{p+1}\left(1-x_{i}\right) \leq F_{p}\left(\frac{p}{p+1}\right) \sum_{i=1}^{n} x_{i}=F_{p}\left(\frac{p}{p+1}\right) .
$$

Thus, we find $C_{p, 2}=C_{p, n}=F_{p}\left(\frac{p}{p+1}\right)=\frac{p^{p}}{(p+1)^{p+1}}$. So we have proved Proposition 1.3.

\section{Remarks}

3.1. In the case where $1<p<2$, we have only obtained upper and lower estimates for the best constant $C_{p, n}$. We believe that (in this case) the following equality holds

$$
C_{p, 2}=F_{p}\left(\frac{p}{p+1}\right)
$$

but we have no proof. We conclude this note by giving another problem generalizing Problem 1.2.

3.2 Problem. Let $n, k$ be positive integers such that $k \leq n$ and $p>0$. Determine (a) the smallest constant $C_{p, n, k}$ such that

$\sum_{1 \leq i_{1}<i_{2}<\ldots<i_{k} \leq n} x_{i_{1}} x_{i_{2}} \ldots x_{i_{k}}\left(x_{i_{1}}^{p}+x_{i_{2}}^{p}+\ldots+x_{i_{k}}^{p}\right) \leq C_{p, n, k}\left(\sum_{i=1}^{n} x_{i}\right)^{p+k}$,

for all non-negative real numbers $x_{1}, x_{2}, \ldots, x_{n}$ satisfying $\sum_{i=1}^{n} x_{i}=$ 1 , and (b) the cases of equality. 


\section{References}

[H] Finbarr Holland, Three problems in one, Irish Mathematical Society Bulletin 43 (1999), 73-78.

Mohamed Akkouchi,

Département de Mathématiques,

Faculté des Sciences-Semlalia,

Université Cadi Ayyad,

Avenue du prince My. Abdellah. B.P. 239,

Marrakech, Morocco

Received on 1 March 2000 and in revised form on 5 December 2000. 